\title{
REMEDIAÇÃO DE EFLUENTES DERIVAdOS DA INDÚSTRIA DE PAPEL E CELULOSE. TRATAMENTO BIOLÓGICO E FOTOCATALÍTICO
}

\author{
P. Peralta-Zamora, E. Esposito, J. Reyes e N. Durán \\ Instituto de Química - UNICAMP - CP 6154 - 13083-970 - Campinas - SP
}

Recebido em 24/4/96; aceito em 16/8/96

\begin{abstract}
REMEDIATION OF EFFLUENTS FROM PAPER AND CELLULOSE INDUSTRY. BIOLOGICAL AND PHOTOCATALYTIC TREATMENT. The contribution of the industrial activities to the environmental contamination phenomena is evident. Great efforts are dedicated to the establishment of methodologies which permits an adequate treatment of the produced effluents, as a manner of minimizing the environmental impact of these wastes. The methodologies based on photocatalytic processes are very promise alternatives, because permits degradation of a great number of chemical substances of high toxic potential, without the use of other chemicals. The present work is an overview about the principal environmental aspects related with the paper and cellulose industry and the main alternatives employed for the reduction of environmental impact produced for its residues. The principal results of the photocatalytic treatment of this kind of effluents using metallic semiconductors is also showed.
\end{abstract}

Keywords: paper and cellulose effluents; biological and photocatalytic treatment; metallic semiconductors.

\section{INTRODUÇÃO}

O desmedido crescimento populacional dos nossos dias, junto com a popularização do modelo consumista gerado nos países do chamado "primeiro mundo", tem propiciado a explosão industrial que caracteriza as últimas décadas. Esta atividade industrial é responsável pela produção de inúmeros produtos de primeira necessidade, razão pela qual tem-se transformado em verdadeiro símbolo das sociedades desenvolvidas. Além de proporcionar alimentos, materiais e serviços, a atividade industrial costuma ser responsabilizada, muitas vezes com justa razão, pelo fenômeno de contaminação ambiental, tema que deixou de ser preocupação exclusiva de cientistas e visionários e transformou-se em clamor geral de uma sociedade que testemunha a deterioração progressiva do planeta.

Embora exista uma preocupação universal por evitar episódios de contaminação ambiental, estes eventos continuam acontecendo, principalmente por que grande parte dos processos produtivos são intrinsecamente poluentes. Muitos estudos, objetivando desenvolver tecnologia capaz de minimizar o volume e a toxicidade dos efluentes industriais, têm sido realizados. Atualmente, por exemplo, existe uma forte tendência nas indústrias de papel e celulose pelo estabelecimento de tecnologias limpas, as quais poderiam permitir, em princípio, atingir o estado de descarga zero ${ }^{1-6}$. Infelizmente, a aplicabilidade deste tipo de sistemas está subordinada ao desenvolvimento de processos modificados e ao estabelecimento de sistemas de reciclagem de efluentes, atividades que implicam tecnologias não universalmente disponíveis. Por este motivo, o estudo de novas alternativas para o tratamento e a remediação dos inúmeros efluentes industriais atualmente produzidos, continua sendo uma das principais armas de combate contra a contaminação antropogênica.

Com este propósito, muitas técnicas têm sido desenvolvidas. Alguns procedimentos clássicos como incineração e procedimentos que envolvem transferência de fases (ex.: ultrafiltração, adsorção, precipitação, e "air-stripping"), mostram valor bastante discutível e custos relativamente elevados. O primeiro ${ }^{65}$, principalmente por que, com certa freqüência, leva à formação de compostos mais tóxicos que o próprio efluente (tipicamente dioxinas), e o segundo ${ }^{66}$, por que não elimina as substâncias contaminantes, apenas as transfere para uma nova fase. Tratamentos baseados em processos biológicos são freqüentemente utilizados, já que permitem a transformação de compostos orgânicos tóxicos em $\mathrm{CO}_{2} \mathrm{e}_{2} \mathrm{O}$, nos processos aeróbios, ou $\mathrm{CH}_{4}$ e $\mathrm{CO}_{2}$, nos anaeróbios; com custos relativamente baixos. Contudo, tratam-se de procedimentos que requerem um longo tempo de operação para que o efluente atinja os padrões exigidos. Novas tendências dentro desta área de pesquisa incluem a utilização direta de enzimas imobilizadas ${ }^{51-53,56}$.

Dentre os novos métodos desenvolvidos com o objetivo de remediar efluentes industriais, destacam-se os processos oxidativos avançados ${ }^{15}$. Estes métodos se fundamentam na geração do radical hidroxila, de características fortemente oxidantes. As vantagens mais significativas deste tipo de procedimentos, estão representadas pela grande eficiência na degradação de compostos orgânicos tóxicos e persistentes (ex.: compostos organoclorados), sem a necessidade de recorrer à utilização de outros oxidantes químicos, mais enérgicos e mais poluentes.

\section{INDÚSTRIA DE PAPEL E CELULOSE, ASPECTOS AMBIENTAIS}

Em 1987 a produção mundial de papel e polpas de madeira foram estimadas em 227 e 50 milhões de toneladas, respectivamente ${ }^{7}$. Diariamente, a indústria de papel e celulose libera mais de 62 milhões de metros cúbicos de efluentes, o que corresponde ao consumo doméstico de água de aproximadamente 200 milhões de pessoas ${ }^{8}$. O impacto ambiental causado por estas descargas líquidas é um problema de caráter grave ${ }^{9}$.

O processo de polpação predominante no mundo é o processo kraft, o qual é responsável pela geração de efluentes com alta demanda bioquímica de oxigênio, turbidez, cor e sólidos suspensos, e baixas concentrações de oxigênio dissolvido ${ }^{10}$. Etapas posteriores de branqueamento, universalmente realizadas através de cloração, levam à formação de um grupo de compostos de estrutura diversa, denominados "cloroligninas"11. Os efluentes resultantes deste processo de branqueamento, são fortemente coloridos e contém muitas substâncias orgânicas, principalmente fenóis clorados, as quais apresentam toxicidade para muitos organismos aquáticos e alta resistência à degradação 
microbiana $^{7-12}$. Mais de 300 compostos orgânicos foram detectados em efluentes de branqueamento, alguns de reconhecido efeito tóxico e/ou genotóxico (ex. fenóis clorados, derivados de catecol e guaiacol, dioxinas, etc. $)^{7}$.

\subsection{Efluentes Gerados Durante Etapas de Polpação e Branqueamento}

$\mathrm{O}$ processo de polpação kraft, realizado com $\mathrm{NaOH}$ e $\mathrm{Na}_{2} \mathrm{~S}$, remove aproximadamente $90 \%$ da lignina presente na madeira, e produz um efluente de alta carga orgânica denominado "licor negro". Este efluente, obviamente alcalino (pH aproximadamente 12.), apresenta alta concentração de espécies fenólicas.

A lignina residual presente nas fibras pode ser eliminada por processos de branqueamento, os quais geralmente são realizados por meio de uma sequiência de estágios de cloração e extração alcalina. Uma seqüência típica de branqueamento está constituída dos estágios seguintes: $\mathrm{Cl}_{2}(\mathrm{C} 1)$, extração alcalina (E1), hipoclorito (H), dióxido de cloro (D1), extração alcalina (E2) e dióxido de cloro (D2).

O efluente derivado da primeira extração alcalina (efluente kraft E1), apresenta um pH entre 9 e 13, e contém altas concentrações de compostos organoclorados (cloroligninas e clorofenóis). A mistura de branqueamento corresponde a uma combinação dos efluentes de cada estágio de branqueamento, enquanto que o efluente final corresponde ao produto do tratamento dos efluentes da etapa de branqueamento, em lagoas aeróbias.

\section{FOTOCATÁLISE HETEROGÊNEA}

A degradação de compostos orgânicos através de fotocatálise heterogênea tem sido bastante documentada ${ }^{13-17}$. Conforme representado na figura 1, quando um semicondutor é exposto a luz ultravioleta, este é promovido a um estado eletronicamente excitado, que se caracteriza pela geração de um par elétronlacuna $\left(\mathrm{e}-, \mathrm{h}^{+}\right)$. As características oxidantes do radical hidroxila gerado por reação da lacuna (equação 1), e o caráter fortemente oxidante da própria lacuna, fazem com que as moléculas orgânicas adsorvidas na superfície da partícula de catalisador possam ser oxidadas até a completa mineralização, através de um processo bastante viável.

$\mathrm{OH}^{-}+\mathrm{TiO}_{2}\left(\mathrm{~h}^{+}\right) \varnothing \mathrm{OH}^{*}+\mathrm{TiO}_{2}$

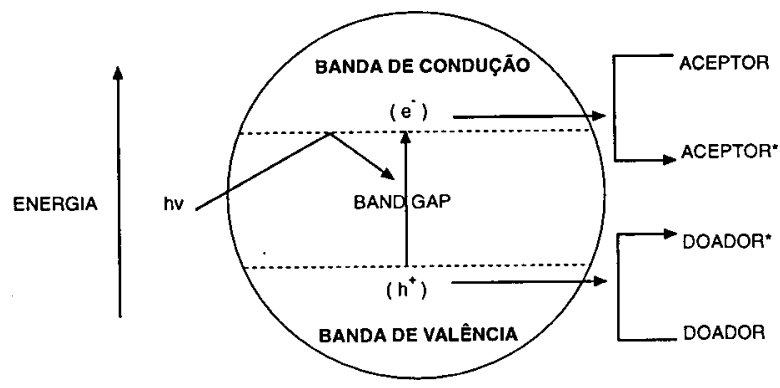

Figura 1. Representação dos princípios energéticos da fotocatálise.

Dentro do contexto da remediação de efluentes derivados da indústria de papel e celulose, a fotocatálise heterogênea tem sido utilizada com sucesso na degradação de celulose ${ }^{18}$, lignina ${ }^{19-21}$, dioxinas $^{22}$, di, tri e tetracloro derivados de metano e etano ${ }^{23,24}$, fenol $^{25}$ e pentaclorofenol ${ }^{26}$; utilizando-se $\mathrm{TiO}_{2}$ como catalisador. Para efluente $\mathrm{kraft}^{27}$ e licor negro de $\mathrm{kraft}^{28}$, no entanto, o catalisador $\mathrm{TiO}_{2}$ não funciona de maneira eficiente. Para este tipo de efluentes, $\mathrm{ZnO}$ mostrou-se muito mais conveniente ${ }^{27,29}$; permitindo, inclusive, a sua utilização como sistema de pré-tratamento para descontaminação biológica de efluentes ${ }^{27,30-35}$.

\subsection{Tratamento Fotocatalítico de Efluentes Derivados da Indústria de Papel e Celulose. Utilização de Óxidos Metálicos Semicondutores nas Formas Livres e Suportada}

A utilização de óxidos semicondutores, como catalisador em processos destinados à degradação de compostos orgânicos de interesse ambiental, tem sido objeto de importantes estudos. As principais limitações na utilização deste tipo de catalisadores $\left(\mathrm{TiO}_{2}, \mathrm{ZnO}, \mathrm{CdS}, \mathrm{Fe}_{2} \mathrm{O}_{3}\right.$, etc.), estão representadas pela importante perda de penetração de luz, num sistema que contém uma fina suspensão de partículas opacas, e pelas dificuldades existentes na etapa de remoção deste material particulado. Para contornar estes inconvenientes, a utilização de catalisadores imobilizados tem-se mostrado como uma boa alternati$\mathrm{va}^{36,37}$. Os suportes utilizados com maior freqüência são: vidro, teflon, materiais poliméricos, sílica, carvão ativado, cerâmicas, aço, etc ${ }^{13,37}$.

Utilizando-se varios óxidos metálicos nas formas livre e suportada, para tratamento fotoquímico de diversos efluentes de indústria papeleira, obtiveram-se os resultados apresentados na tabela $1^{63}$. Observa-se neste conjunto de resultados, uma tendência geral que implica maior eficiência de $\mathrm{TiO}_{2}$ na descoloração de mistura de branqueamento e efluente E1 (efluentes que foram testados em condições ácidas), e eficiência comparável entre $\mathrm{TiO}_{2}$ e $\mathrm{ZnO}$ para licor negro e efluente final (efluente alcalino e ligeiramente alcalino, respectivamente). Em comparação com os catalisadores anteriores, o $\mathrm{ZrO}_{2}$ apresenta resultados pouco expressivos.

Com relação às formas suportadas, observa-se claramente que a eficiência do catalisador é reduzida pela imobilização. Cabe salientar, no entanto, que a quantidade de $\mathrm{ZnO}$ presente nas massas de catalisador suportado utilizadas neste estudo, são significativamente menores que as quantidades de $\mathrm{ZnO}$ livre. Uma comparação adequada do efeito da imobilização na atividade fotocatalítica de $\mathrm{ZnO}$ deveria, necessariamente, considerar massas idênticas do óxido. Contudo, para se atingir a quantidade de catalisador livre, seria necessário utilizar quantidades muito grandes de catalisador imobilizado. Nestas condições, a eficiência do processo diminui radicalmente, em função da perda de eficiência da radiação; este último devido a absorção por parte das grandes quantidades de suporte. $\mathrm{O}$ fato mais importante, em relação à utilização de catalisador suportado, está representado pela verificação da sua atividade fotocatalítica (especialmente interessante no tratamento de efluente final), ainda quando preparado em concentrações bastante reduzidas (1-2\%), e das significativas vantagens que a imobilização permite nas operações de remoção do catalisador.

Em relação ao tipo de suporte, observa-se que a porcelana mostra importantes graus de adsorção, superiores em todos os casos aos apresentados pela sílica. Este fato, provavelmente devido a maior atividade fisica e química da superfície da porcelana, mostra-se altamente inconveniente, já que impede, ou pelo menos dificulta, os processos fotocatalíticos.

Estudando-se a degradação dos compostos orgânicos presentes nos efluentes estudados ${ }^{63}$, por meio da avaliação de descoloração, redução de fenóis totais (compostos que caracterizam este tipo de efluentes) e redução de carbono orgânico total, obtiveram-se os resultados sumarizados na tabela 2. Estes resultados indicam que para o efluente final ambos catalisadores na forma livre permitem importantes graus de descoloração e de redução de formas fenólicas, aproximadamente $80 \%$, para tempos de irradiação não superiores a $60 \mathrm{~min}$. A utilização de $\mathrm{ZnO}$ imobilizado em sílica permite menor redução destes parâmetros, no entanto, a tendência observada sugere a possibilidade de se atingir eficiência similar, para maiores tempos de irradiação. Para licor negro os catalisadores livres mostram uma alta eficiência de descoloração (aproximadamente 80\%) e redução quase completa da concentração de formas fenólicas. A eficiência do catalisador imobilizado mostra-se alta para a redução 
Tabela 1. Descoloração de efluentes papeleiros com diversos catalisadores. Volume de efluente: $25 \mathrm{~mL}$; Tempo de irradiação: 60 min; Oxigênio: $200 \mathrm{mLmin}^{-1}$; Massa de catalisador: $\mathrm{TiO}_{2}: 25 \mathrm{mg}$; ZnO: $25 \mathrm{mg}$; ZnO-porcelana: $200 \mathrm{mg}$; ZnO-sílica: $150 \mathrm{mg}$; $\mathrm{ZrO}_{2}$-sílica: $100 \mathrm{mg}$.

\begin{tabular}{|c|c|c|c|}
\hline EFLUENTE & CATALISADOR & $\begin{array}{c}\text { DESCOLORAÇÃ }^{1} \\
(\%)\end{array}$ & $\begin{array}{c}\mathrm{ADSORÇ}^{\mathrm{A} \mathrm{O}^{2}} \\
(\%)\end{array}$ \\
\hline $\begin{array}{l}\text { KRAFT E1 } \\
(\mathrm{pH}: 5,0)\end{array}$ & $\begin{array}{c}\mathrm{TiO}_{2} \text { livre } \\
\mathrm{ZnO} \text { livre } \\
\mathrm{ZnO} \text {-sílica } \\
\text { ZnO-porcelana } \\
\mathrm{ZrO}_{2} \text {-sílica }\end{array}$ & $\begin{array}{c}60 \\
50 \\
- \\
- \\
-45\end{array}$ & $\begin{array}{c}38 \\
23 \\
- \\
- \\
-04\end{array}$ \\
\hline $\begin{array}{l}\text { LICOR NEGRO } \\
\quad(\mathrm{pH}: 9,0)\end{array}$ & $\begin{array}{c}\mathrm{TiO}_{2} \text { livre } \\
\mathrm{ZnO} \text { livre } \\
\mathrm{ZnO} \text {-sílica } \\
\text { ZnO-porcelana } \\
\mathrm{ZrO}_{2} \text {-sílica }\end{array}$ & $\begin{array}{l}75 \\
74 \\
23 \\
19 \\
31\end{array}$ & $\begin{array}{l}01 \\
00 \\
03 \\
25 \\
04\end{array}$ \\
\hline $\begin{array}{c}\text { MISTURA DE } \\
\text { BRANQUEAMENTO } \\
(\mathrm{pH}: 2,5)\end{array}$ & $\begin{array}{c}\mathrm{TiO}_{2} \text { livre } \\
\mathrm{ZnO} \text { livre } \\
\mathrm{ZnO} \text {-sílica } \\
\text { ZnO-porcelana } \\
\mathrm{ZrO}_{2} \text {-sílica }\end{array}$ & $\begin{array}{l}66 \\
27 \\
34 \\
10 \\
20\end{array}$ & $\begin{array}{l}49 \\
13 \\
02 \\
14 \\
02\end{array}$ \\
\hline $\begin{array}{l}\text { EFLUENTE FINAL } \\
(\mathrm{pH}: 7,6)\end{array}$ & $\begin{array}{c}\mathrm{TiO}_{2} \text { livre } \\
\mathrm{ZnO} \text { livre } \\
\mathrm{ZnO} \text {-sílica } \\
\mathrm{ZnO} \text {-porcelana } \\
\mathrm{ZrO}_{2} \text {-sílica }\end{array}$ & $\begin{array}{l}95 \\
82 \\
55 \\
41 \\
45\end{array}$ & $\begin{array}{l}-27 \\
36 \\
01 \\
36 \\
04\end{array}$ \\
\hline
\end{tabular}

(1) Descoloração observada no tratamento fotocatalítico.

(2) Descoloração alcançada por adsorção na superfície do catalisador (procedimento realizado na ausência de irradiação).

Tabela 2. Redução de cor, fenóis totais e carbono orgânico total (TOC) nos efluentes, após tratamento fotocatalítico. Volume de efluente: $25 \mathrm{~mL}$; Tempo de irradiação: $60 \mathrm{~min}$; Oxigênio: $200 \mathrm{mLmin}^{-1}$; Massa de catalisador: $\mathrm{TiO}_{2}: 25 \mathrm{mg}$; $\mathrm{ZnO}$ : $25 \mathrm{mg}$; ZnO-sílica: $150 \mathrm{mg}$.

\begin{tabular}{lccc}
\hline EFLUENTE & \multicolumn{3}{c}{ CATALISADOR } \\
& $\mathrm{TiO}_{2}$ & $\mathrm{ZnO}$ & ZnO-sílica \\
\hline Licor negro & & & \\
Redução de cor & 75 & 74 & 23 \\
Redução de fenóis & 100 & 100 & 60 \\
$\begin{array}{l}\text { Redução de TOC } \\
\text { (\%) }\end{array}$ & - & - & - \\
Efluente final & & & \\
Redução de cor & 95 & 82 & 55 \\
Redução de fenóis & 83 & 77 & 44 \\
Redução de TOC & 50 & 10 & 05 \\
(\%) & & & \\
Mistura de branq. & & 27 & 34 \\
Redução de cor & 60 & 10 & 00 \\
$\begin{array}{l}\text { Redução de fenóis } \\
\text { Redução de TOC }\end{array}$ & 00 & 20 & 05 \\
$(\%)$ & & & \\
\hline
\end{tabular}

* O teor de fenóis totais aumenta significativamente nos primeiros 30 min de irradiação. Ultrapassado este tempo, a concentração de formas fenólicas diminui gradativamente até atingir, após $60 \mathrm{~min}$, valores iguais ou levemente menores que os iniciais.

de fenóis totais, enquanto que seu efeito na descoloração é pouco significativo.

No tratamento fotocatalítico de mistura de branqueamento obtém-se resultados algo diferentes. A redução da cor é bastante alta para $\mathrm{TiO}_{2}$ livre e significativamente inferior para $\mathrm{ZnO}$ nas formas livre e imobilizada. O comportamento dos fenóis totais, por outro lado, mostra-se totalmente anômalo. Resulta difícil explicar um aumento tão significativo da concentração de formas fenólicas, nos primeiros instantes de irradiação. As explicações poderiam ser formuladas em termos de arranjo de grupos fenólicos, fato que poderia ser favorecido pelo processo fotolítico e que levaria à formação de espécies sensíveis à reação de Folin-Ciocalteus ${ }^{64}$ (reação em que fundamenta-se a metodologia de determinação de fenóis totais); ou como real formação de espécies fenólicas por ataque do radical hidroxila. O mais importante é que a concentração de espécies fenólicas tende a diminuir para maiores tempos de irradiação; podendo, provavelmente, ser eliminada totalmente com a extensão do processo fotocatalítico ${ }^{63}$.

A redução do teor de carbono orgânico total durante o processo fotocatalítico é apresentada na tabela 2. Observa-se claramente que a degradação de compostos orgânicos, verificada pela diminuição de cor e fenóis totais, não reflete importantes graus de mineralização. Isto é, o processo de degradação acontece, mas não de maneira que permita a completa eliminação da carga orgânica do efluente. As significativas variações de cor e fenóis totais demonstram que o processo fotocatalítico promove alterações na estrutura das substâncias orgânicas, no entanto, com os tempos de irradiação praticados, tais reações devem levar à formação de intermediários e não à completa degradação. Esta observação é de grande importância, já que não é incomum o fato de reações fotocatalíticas levarem, nos instantes iniciais, à formação de espécies mais tóxicas que as originalmente presentes no efluente. Por este motivo se faz fundamental a realização de testes de toxicidade de intermediários; se a toxicidade é demonstrada, a extensão do processo fotocatalítico até completa mineralização se faz fundamental.

\subsection{Comentários Finais Sobre o Tratamento Fotocatalítico}

Os resultados apresentados demonstram claramente a viabilidade do processo fotocatalítico, para a remediação dos efluentes derivados da indústria de papel e celulose. Características como cor e fenóis totais, podem ser reduzidas com extrema eficiência, utilizando-se tempos de irradiação relativamente 
pequenos. A redução de carbono orgânico total, que atesta a mineralização das espécies orgânicas, não atinge graus significativos; no entanto, os resultados sugerem que com maiores tempos de tratamento, a completa mineralização seja possível.

O efeito fotocatalítico de catalisadores suportados é evidente. Embora a eficiência do processo diminua, inúmeras vantagens de ordem prática justificam a sua utilização.

\section{MÉTODOS BIOLÓGICOS PARA REMEDIAÇÃO DE EFLUENTES DERIVADOS DA INDÚSTRIA DE PAPEL E CELULOSE}

As técnicas universalmente utilizadas na descontaminação de efluentes industriais são as de lagoas aeróbicas e lodos ati$\operatorname{vados}^{7}$. Em geral, tratamentos anaeróbicos são pouco utilizados. Interessantes revisões sobre este tema já têm sido realizadas ${ }^{38,39}$.

Normalmente, a toxicidade aguda é eliminada nas lagoas aeróbicas; no entanto, ligninas cloradas de elevada massa molar são resistentes à degradação por consórcios de bactérias isoladas destas lagoas ${ }^{40}$. Estes aspectos têm sido discutidos por Roy-Arcand e Archibald ${ }^{41}$.

O sistema de lodos permite uma redução mais significativa de compostos clorados $(40 \%)$, em comparação aos resultados conseguidos em lagoas aeróbicas $(25 \%)^{42}$. O processo consiste, basicamente, na agitação dos efluentes na presença de microrganismos e oxigênio atmosférico, durante o tempo necessário para metabolizar e flocular uma grande parte da matéria orgânica. Embora este tipo de tratamento mostre-se bastante eficiente, os lodos ativados apresentam o grande inconveniente de formar quantidades exageradas de lodo. Atualmente existem programas que consideram a utilização destes lodos como fertilizantes para solo (ex. Riocell Celulose, Porte Alegre - RS).

A atual tendência no tratamento de efluentes derivados da indústria de papel e celulose, está representada pela utilização de processos anaeróbicos-aeróbicos, alternados. Este sistema aumenta significativamente a eficiência do processo, o que permite a redução do tamanho das estações e dos tempos de residência $^{42-44}$. Na Finlândia, por exemplo, está sendo aplicado com grande sucesso um tratamento combinado deste tipo. Este procedimento, denominado Enso-Fenox, permite em 7 horas de operação a mesma redução de halogênios adsorvíveis alcançada em 7 dias de tratamento em lagoas aeróbicas; no entanto, compostos de alta massa molar não são degradados ${ }^{44}$.

Uma alternativa que tem-se mostrado muito interessante, motivo pelo qual está sendo submetida a intensa avaliação, é a utilização de biorreatores operados com fungos. Sistemas que operam com fungos imobilizados em diversos suportes têm sido utilizados com grande sucesso na degradação de clorofenóis ${ }^{45-50}$.

Recentes publicações têm reportado alta eficiência de algumas enzimas no tratamento de efluentes (ex.: lacases e peroxidases). Entretanto, os estudos sugerem que aplicações industriais, necessariamente devem envolver a utilização de enzimas imobilizadas. Bons resultados têm sido obtidos na imobilização de peroxidase de raiz forte e lignina peroxidase (de Chrysonilia sitophila) em Sepharose ${ }^{51,52}$ e lacase em alginato ${ }^{53}$; este último estudo reporta eficiência significativamente maior na descoloração de efluentes fenólicos, quando se utiliza enzima imobilizada. Peroxidase livre ${ }^{54,55}$ e imobilizada ${ }^{56}$ têm-se mostrado eficientes no tratamento de fenóis e clorofenóis. Estudos mecanísticos com cloroperoxidases, peroxidase de rabanete, lignina peroxidase, Mn-peroxidase e lacase; também têm sido realizados ${ }^{57}$.

Utilizando-se um biorreator de leito fixo (packed bed) combinado com uma alça de ar (air-lift) e o fungo $P$. chrysosporium, foi avaliada a remediação de efluente Kraft. Com tempos de residência de 5 dias, obtiveram-se descoloração de $70 \%$, remoção de fenóis de $64 \%$ e redução de DQO de $50 \%{ }^{58}$. Utilizando-se cepas pré-selecionadas de Lentinula edodes ${ }^{31,32,59}$ e um biorreator do mesmo tipo anterior, obtiveram-se resultados similares de descoloração e redução de fenóis, com uma maior eficiência na redução do DQO $(67 \%)^{60}$. Lignina peroxidase de Chrysonilia sithophila foi imobilizada em sílica e aplicada com sucesso no tratamento de efluente $\mathrm{kraft}^{61}$.

Métodos fotoquímicos têm sido utilizados como sistema de pré-tratamento para descontaminação biológica de efluentes $27,30,33-35,62$.

\section{CONCLUSÕES}

A indústria de papel e celulose tem sido objeto de fortes pressões para investir em tecnologias que permitam diminuir o impacto ambiental causado por suas atividades. A utilização de processos de branqueamento isentos de cloro (ECF: Elemental Chlorine Free e TCF: Totally Chlorine Free) tem contribuído significativamente com a diminuição de compostos organoclorados. Por outro lado, estudos direcionados ao estabelecimento de processos de recirculação de efluentes, os quais finalmente objetivam o estabelecimento de um ciclo fechado, muito tem contribuído á diminuição do volume dos efluentes produzidos. Estudos são realizados para estabelecer as condições que permitam alcançar o estado de "descarga zero". Atingir este estado, no entanto, implica a utilização de tecnologias que não são de domínio universal. Para se resolver o problema ambiental do presente momento, portanto, se faz necessário o desenvolvimento de metodologias que permitam remediar os efluentes produzidos. Com este objetivo muitas alternativas têm sido estudadas, entre as que se destacam a fotocatálise heterogênea, utilizandose semicondutores metálicos, e os métodos biológicos, representados principalmente pela utilização de fungos e enzimas. Inúmeros estudos continuam sendo realizados, visando a otimização das metodologias de tratamento propostas e o desenvolvimento de novos processos, mais eficientes, rápidos e econômicos.

\section{REFERÊNCIAS}

1. Evans, T.; Sweet, B.; Manoluscu, D. and Mason, J.; Pulp Paper Can. 1995, 96, 60.

2. Heatley, W. R. and Barfield, M.; Tappi J. 1995, 78, 197.

3. Kenny, R.; Yampolsky, M. and Goncharov, A.; Pulp Paper Can. 1995, 96, 26.

4. Odendhal, S.; Pulp Paper Can. 1994, 95, 30.

5. Sundquist, J.; Paperi Ja Puu-Paper and Timber. 1994, $76,22$.

6. Williamson, P. N.; Pulp Paper Can. 1994, 95, 9.

7. Durán, N. and Esposito, E.; Quim. \& Ind. 1993, 2, 17.

8. Sant'Anna, G. L.; Proc 2nd Braz. Symp. Chem. Lignins and Other Wood Comp. 1992, 3, 297.

9. Ribó, J. M.; Afinidad. 1992, 437, 7.

10. Araújo, R. P. A.; Bertoletti, E.; Ramos, M. L. L. C.; Gherardi-Goldstein, E. and Martins, M. H. R. B.; Cien. e Cult. 1991, 43, 299.

11. Kirkpatrick, N.; Wat. Sci. Technol. 1991, 24,75.

12. Caron, R. J. and Reeve, D. W.; Pulp Paper Can. 1992, 93,24

13. Ollis, D. F.; Pelizzetti, E. and Serpone, N.; Environ. Sci. Technol. 1991, 25, 1253.

14. Herrmann, J. M.; Guillard, C. and Pichat, P.; Catalysis Today 1993, 17, 20.

15. Legrini, O.; Oliveros, E. and Braun, A. M.; Chem. Rev. 1993, 93, 671 .

16. Linsebigler, A. L.; Guangquan, L. and Yates, Jr. T.; Chem. Rev. 1995, 95, 735

17. Hoffmann, M.; Martin, S. T.; Choi, W. and Bahnemann, W.; Chem. Rev. 1995, 95, 69.

18. Vesely, M.; Ceppan, M.; Brezova, V. and Lapcik, L.; J. Photochem. Photobiol. A. Chem. 1991, 61, 399.

19. Kobayakawa, K.; Sato, S.; Nakamura, S. and Fujishima, A.; Bull. Chem. Soc. Jpn. 1989, 62, 3433. 
20. Ohnishi, H.; Matsumura, M.; Tsubomura, H. and Iwasaki, M.; Ind. Eng. Chem. Res. 1989, 28, 719.

21. Zeltner, W. A.; Hill, C. G. and Anderson, M. A.; CHEMTEC. 1993, 23, 21.

22. Friesen, K. J; Muir, D. C. G. and Webster, G. R. B.; Environ. Sci. Technol. 1990, 24, 1739.

23. Hisanaga, T.; Harada, K. and Tanaka, K.; J. Photochem. Photobiol. A: Chem. 1990, 54, 113.

24. Glaze, W. A.; Kenneke, J. F. and Ferry, J. L.; Environ. Sci. Technol. 1993, 27, 177.

25. Augugliaro, V.; Inglese, F.; Palmisano, M. and Schiavelo, M.; Chem. Biochem. Eng. Q. 1992, 6, 63.

26. Mills, G. and Hoffmann, R.; Environ. Sci. Technol. 1993, 27,1681

27. Durán, N.; Dezotti, M. and Rodriguez, J.; J. Photochem. Photobiol. A. Chem. 1991, 62, 269.

28. Mansilla, H. D.; Villaseñor, J. and Maturana, G. J.; J. Photochem. Photobiol. A: Chem. 1994, 78, 267.

29. Reyes, J.; Mansilla, H. D.; Villaseñor, J. and Durán, N.; 3th Braz. Symp. Chem. Lignins and Other Wood Comp. Abstr. WAP-32, 1993.

30. Esposito, E.; Canhos, V. P. and Durán, N.; Proc. 2nd. Braz. Symp. Chem. Lignins and Other Wood Comp. 1992, 3, 356.

31. Esposito, E.; Durán, N.; Freer, J.; Baeza, J. and Innocentini-Mei, L. H.; Proc. CHEMPOR-93, Intern. Chem. Eng. Conf. 1993, 201.

32. Esposito, e.; Innocentini-Mei, L. H.; Ferraz A.; Canhos, V. P. and Durán, N.; J. Biotechnol. 1993, 29, 219.

33. Durán, N.; Proc. 2nd. Braz. Symp. Chem. Lignins and Other Wood Comp. 1992, 3, 323.

34. Durán, N.; Esposito, E. and Canhos, V. P.; In Cellulosics: Pulp, Fibre and Environmental Aspects. In Polymer Sci. and Technol. 1993, 73, 493.

35. Durán, N.; Esposito, E.; Innocentini-Mei, L. H. and Canhos, V. P.; Biodegradation 1994, 5, 13.

36. Sabate, J.; Anderson, M. A.; Aguado, M. A.; Giménez, J.; Cervera-March, S. and Hill, C. G.; J. Mol. Catal. 1992, $71,57$.

37. Bideau, M.; Claudel, B.; Dubien, C.; Faure, L. and Kazouan, H.; J. Photochem. Photobiol. A: Chem. 1995, 91, 137.

38. Lee, J. W.; Petersen, D. L. and Stickney, A. R.; Environ. Paper 1989, 8, 73.

39. Rintala, J. A. and Puhakka, J. A.; Bioresource Technol. 1994, 47, 1 .

40. Eriksson, K. E. and Kolar, M. C.; Environ. Sci. Technol. 1985, 19, 1086

41. Roy-Arcad, L. and Archibald, F. S.; Wat. Res. 1993, 27, 873.
42. Eriksson, K. E.; Proc. 2nd. Braz. Symp. Chem. Lignins and Other Wood Comp. 1992, 3, 274.

43. Fairbanks, M.; Quim. Derivado 1992, 291, 16.

44. Ek. M. and Eriksson, K. E.; Proc. VVT Symp. 102, NonWater Technol. 1989, 1, 435.

45. Lewandowski, G.A.; Armenante, P. M. and Park, D.; Wat. Res. 1990, 24, 75.

46. Prouty, A. L.; Appl. Microbiol. Biotechnol. 1990, 32, 490.

47. Prasad, D. Y. and Joyce, T. W.; TAPPI J. 1991, 74, 165.

48. Lankinen, V. P.; Inkeroinen, M. M.; Pelline, J. and Hattaka, A. I.; Wat. Sci. Technol. 1991, 24, 189.

49. Anselmo, A. M. and Novais, J. M.; Wat. Sci. Tech. 1992, $25,161$.

50. Anselmo, A. M. and Novais, J. M.; Biotechnol. Lett. 1992, $14,239$.

51. Ferrer, I.; Dezotti, M. and Durán, N.; Biotechnol. Lett. 1991, 13, 577.

52. Ferrer, I.; Esposito, E. and Durán, N.; Enzyme Microb. Technol. 1992, 14, 402.

53. Davis, S. and Burns, R. G.; Biodegradation 1994, 5, 13.

54. Nicell, J. A.; Bewtra, J. K; Biswas, N. and Taylor, E.; Wat. Res. 1993, 27, 1629.

55. Nakamoto, S. and Machida, N.; Wat. Res. 1992, 26, 49.

56. Siddique, M. H.; Pierre, C. C.; Biswas, N.; Bewtra, J. K. and Taylor, K. E.; Wat. Res. 1993, 27, 883.

57. Aitken, M. D.; Massey, I. J.; Chen, T. and Heck, P. E.; Wat. Res. 1994, 28, 1879.

58. Cammarota, M. C. and Sant'Anna, G. L.; Environ. Technol. 1992, 13, 65.

59. Esposito, E.; Canhos, V. P. and Durán, N.; Biotechnol. Lett. 1991, 13, 571.

60. Durán, N.; Esposito, E.; Baeza, J.; Freer, J. and Rodriguez, J.; Proc. I Taller de Biotecnologia Ambiental. Universidad de Concepción (Chile), 1992.

61. Dezotti, M.; Innocentini-Mei, L. H. and Durán, N.; J. Biotechnol. 1995, 43, 161.

62. Esposito, E.; Proc. I Seminário Nacional de Tecnologia Enzimática, ENZITEC-93. 1993, 30.

63. Peralta-Zamora, P.; Pelegrini, R.; Frire Jr, M.; Reyes, J. and Durán, N. Wat. Res. 1996, submetido para publicação.

64. APHA. Standard Methods for Examination of water and Wastewater, 17 ed. New York, Amer. Publ. Health Ass. No 5550. 1989.

65. Manahan, S. C.; Environmental Chemistry. Lewis Publishers, Boca Raton, 1994. 6ta Edição, p.210.

66. Suri, R. P. S.; Liu, J.; Hand, D. W.; Crittenden, J. C. Perram, D. L. and Mullins, M. E.; Wat. Environ. Res. 1993, 65, 665 . 